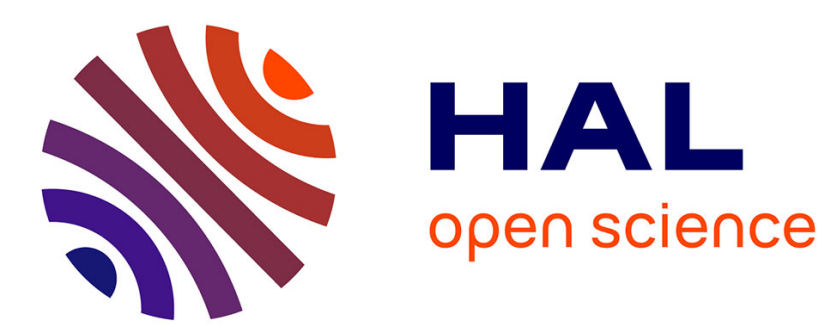

\title{
Thermoacoustic instabilities: Should the Rayleigh criterion be extended to include entropy changes?
}

Franck Nicoud, Thierry Poinsot

\section{To cite this version:}

Franck Nicoud, Thierry Poinsot. Thermoacoustic instabilities: Should the Rayleigh criterion be extended to include entropy changes?. Combustion and Flame, 2005, 142 (1-2), pp.153-159. 10.1016/j.combustflame.2005.02.013 . hal-00908285

\section{HAL Id: hal-00908285 \\ https://hal.science/hal-00908285}

Submitted on 22 Nov 2013

HAL is a multi-disciplinary open access archive for the deposit and dissemination of scientific research documents, whether they are published or not. The documents may come from teaching and research institutions in France or abroad, or from public or private research centers.
L'archive ouverte pluridisciplinaire HAL, est destinée au dépôt et à la diffusion de documents scientifiques de niveau recherche, publiés ou non, émanant des établissements d'enseignement et de recherche français ou étrangers, des laboratoires publics ou privés. 


\title{
Fluctuation energies and stability criteria for thermoacoustic instabilities: is the Rayleigh criterion right ?
}

\author{
Nicoud F. ${ }^{\mathrm{a}}$ and Poinsot T. ${ }^{\mathrm{b}, *}$ \\ ${ }^{a}$ Université Montpellier II, I3M, Place E. Bataillon, 34095 Montpellier, France \\ ${ }^{\mathrm{b}}$ Institut de Mécanique des Fluides de Toulouse, Avenue C. Soula, 31400 \\ Toulouse, France
}

\begin{abstract}
The Rayleigh criterion (which measures the correlation between pressure and heat release) is the standard tool used to investigate and predict combustion instabilities both in experimental and numerical studies. However, the Rayleigh term is just one of the terms appearing in the acoustic energy equation. The recent development of Large Eddy Simulations (LES) for combustion chambers allows to completely close the budget and analyze all terms in this equation. This task leads to unexpected difficulties and requires some basic work since multiple definitions of the energy of fluctuations in a reacting compressible flow can be derived. The objective of this paper is to revisit the theoretical derivations of the fluctuation energy equations. Two forms of energy are defined: the first one is the classical acoustic energy (AE) introduced by various authors $[1-3]$. The second one is the fluctuation energy (FE) presented by $\mathrm{Chu}$ [4]. Both equations are re- derived in a compact manner starting from full non-linear forms. It is shown that the classical Rayleigh criterion naturally appears as the source term of the $\mathrm{AE}$ equation while the $\mathrm{FE}$ form leads to a different criterion stating that temperature and heat release must be in phase for the instability to be fed by the flame/acoustics coupling. The FE form also integrates the fluctuations of three variables (pressure, velocity and entropy) while the $\mathrm{AE}$ form uses only pressure and velocity perturbations. It is shown that only the FE form should be used in flames, in contradiction with many present studies performed for combustion instabilities.
\end{abstract}

Key words: Fluctuation energy, Rayleigh criterion, Large Eddy Simulations

* Corresponding author. Tel: +33-5-61-19-30 34; fax: +33-5-61-19-3030.

Email address: poinsot@cerfacs.fr (Poinsot T.). 


\section{Introduction}

It has been known for a long time that the coupling between acoustic waves and flames in industrial systems can lead to high amplitude instabilities [5, 6, 3]. In addition to inducing oscillations of all physical quantities (pressure, velocities, temperature, etc ...), these instabilities can increase the amplitude of the flame motion and, in extreme cases, destroy part of the burner. A commonly used criterion for assessing the stability of a combustor is the Rayleigh criterion [7], which states that if pressure and heat release fluctuations are in phase, the instability is fed by the flame/acoustics coupling. Formally, this criterion may take the following form:

$$
\iiint_{\Omega} p_{1} q_{1} d \Omega>0
$$

where $p_{1}$ and $q_{1}$ stand for pressure and heat release fluctuations respectively and $\Omega$ is the flow domain. The sign of the above integral may change with the phase of the oscillation and Eq. 1 is often integrated over a period to characterise the stability of the system at a given frequency.

The validity of the Rayleigh criterion can be discussed by introducing a more general analysis tool: the budget of fluctuation energy. Such budgets require the analysis of acoustic quantities such as acoustic fluxes through boundaries and are impossible to construct experimentally. However, recent Large Eddy Simulation tools $[8-11]$ have opened new possibilities in this field by giving access to all unsteady variables at any grid point during combustion oscillations. A better understanding of the underlying physics can be obtained by using the fluctuation energy equation for analysing the LES (Large Eddy Simulation) results rather than the Rayleigh criterion.

The objective of this paper is to present fundamental derivations of the fluctuation energy equation and to discuss them in the framework of thermoacoustic instabilities. A long-term motivation of this work is to provide the theoretical basis for LES analysis and understanding of combustion instabilities: by closing the fluctuation energy equation in a given combustor, it is expected to achieve progress similar to the landmark work performed for turbulence modelling where the budgets of kinetic energy near walls have allowed an understanding of turbulent phenomena which was out of reach before $[12,13]$. The first step towards this ambitious goal is to derive the correct energy equation and more importantly, to determine which energy should be used to measure fluctuations in a reacting compressible flow: this apparently rarely studied topic is the objective of the present work. 
Section 2 first shows how the classical acoustic energy (AE) equation is obtained by linearizing a global energy equation directly deduced from the Navier-Stokes equations and how the Rayleigh criterion enters this budget as one of the source terms. The validity of the Rayleigh criterion is then discussed in terms of energy budget. While the AE equation of Section 2 has been discussed and used by various authors, Section 3 actually shows that this is not the adequate form to be used in reacting flows and proposes an extended formulation. This formulation, called here fluctuation energy equation (FE equation) was first introduced by $\mathrm{Chu}$ [4] but is obviously not very known in the combustion community: it accounts for entropy fluctuations and leads to a new criterion for thermoacoustic instabilities. It is rederived in a more compact way here and corrected for terms missing in Chu's derivation in Section 3.

\section{The acoustic energy (AE) equation}

The derivation leading to the classical form of the acoustic energy equation usually starts from the linearized equations for density and velocity and combines them to form an energy $[4,1,3,2]$. Here a different path is followed: a non-linear equation for "energy" is first derived from the Navier-Stokes equations and is linearized afterwards.

\subsection{A non linear "energy" equation in reacting flows}

To simplify the derivation, all species are supposed to share the same molar weight and constant heat capacities. This assumption is not necessary to derive the generalized acoustic energy equation although it makes the algebra simpler. It is valid for air flames but must be revisited for the case of $\mathrm{H}_{2}-\mathrm{O}_{2}$ mixtures for example. In what follows, $D / D t$ stands for the particular (total) derivative while $\rho, \vec{u}, T, P$ and $s$ are the density, velocity, temperature, pressure and entropy per unit mass of mixture respectively. $C_{p}$ and $C_{v}$ are the usual heat capacity (per unit mass) at fixed pressure and volume respectively. Moreover, $\overrightarrow{\vec{\tau}}$ is the stress tensor whose components are $\tau_{i j}=\mu\left(\partial u_{i} / \partial x_{j}+\partial u_{j} / \partial x_{i}\right)-2 / 3 \delta_{i j} \operatorname{div}(\vec{u})$ and $\mu$ is the dynamic viscosity. $\lambda$ is the heat diffusivity and $\gamma=C_{p} / C_{v}$ and $r=C_{p}-C_{v}$ are the mass heat capacities ratio and difference. Note that the viscous terms (molecular diffusion of momentum and heat) are kept in the present analysis although they are usually neglected for the analysis of acoustic perturbations. The reason is that their inclusion leads to a simple justification for the extended fluctuation energy as shown in section 3. They are neglected in the different stability criteria discussed throughout the paper. 
Under the above assumptions, the momentum equation reads:

$$
\rho \frac{D \vec{u}}{D t}=-\vec{\nabla} P+\vec{\nabla} \cdot \overrightarrow{\vec{\tau}}
$$

Taking the dot product of Eq. 2 with $\vec{u}$, one obtains directly:

$$
\rho \frac{D \vec{u}^{2} / 2}{D t}+\nabla \cdot(P \vec{u})=P \nabla \cdot \vec{u}+\vec{u} \cdot(\vec{\nabla} \cdot \overrightarrow{\vec{\tau}})
$$

In order to obtain an energy-like term for pressure at the LHS, it is convenient to use the transport equation for pressure in order to express the pressure dilatation term in Eq. 3. This equation can be obtained using the sensible energy equation [3]:

$$
\rho \frac{D e_{s}}{D t}=-P \nabla \cdot \vec{u}+q+\nabla \cdot(\lambda \vec{\nabla} T)+\overrightarrow{\vec{\tau}}: \overrightarrow{\vec{\nabla}} \vec{u}
$$

together with the continuity equation $D \rho / D t=-\rho \nabla \cdot \vec{u}$ and the classical relation $\rho e_{s}=P /(\gamma-1)$ valid for perfect gas. It reads:

$$
\frac{D P}{D t}=-\gamma P \nabla \cdot \vec{u}+(\gamma-1)(q+\nabla \cdot(\lambda \vec{\nabla} T)+\overrightarrow{\vec{\tau}}: \overrightarrow{\vec{\nabla}} \vec{u})
$$

where $q$ is the rate of heat release by unit of volume. Injecting Eq. 5 into Eq. 3 and introducing the speed of sound $c=\sqrt{\gamma P / \rho}$ yields:

$$
\begin{aligned}
\rho \frac{D \vec{u}^{2} / 2}{D t}+ & \frac{1}{\rho c^{2}} \frac{D P^{2} / 2}{D t}+\nabla \cdot(P \vec{u})= \\
& \frac{\gamma-1}{\gamma}(q+\nabla \cdot(\lambda \vec{\nabla} T)+\overrightarrow{\vec{\tau}}: \overrightarrow{\vec{\nabla}} \vec{u})+\vec{u} \cdot(\vec{\nabla} \cdot \overrightarrow{\vec{\tau}})
\end{aligned}
$$

The LHS of this equation is formally close to the classical equation for the acoustic energy. One should stress however that it is exact, non-linear and remains valid for any fluctuations of the pressure and velocity fields.

\subsection{Linearization and acoustic energy}

Eq. 6 can be linearized as follows: consider the simple case of small amplitude fluctuations (index 1) super-imposed to a zero Mach number mean flow (index $0)$. The instantaneous pressure, density and velocity fields can then be written as $P=p_{0}+p_{1}, \rho=\rho_{0}+\rho_{1}$ and $\vec{u}=\vec{u}_{1}$ where $p_{1} \ll p_{0}, \rho_{1} \ll \rho_{0}$ and $\vec{u}_{1}^{2} \ll c_{0}^{2}$, 
where $c_{0}=\sqrt{\gamma P_{0} / \rho_{0}}$ is the mean speed of sound. For simplicity, the temporal fluctuations of the diffusivity, viscosity and heat capacities are neglected. Note that the zero Mach number assumption for the mean flow implies $\vec{\nabla} p_{0}=0$ (from Eq. 2) and $q_{0}+\nabla \cdot\left(\lambda \vec{\nabla} T_{0}\right)=0$ (from Eq. 4 ). It also implies that the approximation $D / D t \approx \partial / \partial t$ holds for any fluctuating quantity since, with $u_{0}=0$, the non linear convective term is always of second order. Thus Eq. 5 becomes an equation for the pressure fluctuations :

$$
\frac{D p_{1}}{D t}=-\gamma P \nabla \cdot \vec{u}_{1}+(\gamma-1)\left(q_{1}+\nabla \cdot\left(\lambda \vec{\nabla} T_{1}\right)+\overrightarrow{\vec{\tau}}_{1}: \overrightarrow{\vec{\nabla}} \vec{u}_{1}\right)
$$

With $P=p_{0}+p_{1}$, it is clear that $P^{2}=p_{0}^{2}+2 p_{0} p_{1}+p_{1}^{2}$ and an estimation of $D\left(P^{2} / 2\right) / D t=p_{0} D p_{1} / D t+D\left(p_{1}^{2} / 2\right) / D t$ is:

$$
\begin{aligned}
\frac{D P^{2} / 2}{D t} \simeq & \frac{\partial p_{1}^{2} / 2}{\partial t}-\gamma P p_{0} \nabla \cdot \vec{u}_{1} \\
& +p_{0}(\gamma-1)\left(q_{1}+\nabla \cdot\left(\lambda \vec{\nabla} T_{1}\right)+\overrightarrow{\vec{\tau}}_{1}: \overrightarrow{\vec{\nabla}} \vec{u}_{1}\right)
\end{aligned}
$$

This equation is valid to third order because $\vec{u}_{1} \cdot \vec{\nabla} p_{1}^{2}$ was neglected in front of $\partial p_{1}^{2} / \partial t$. It further implies that :

$$
\begin{aligned}
\frac{1}{\rho c^{2}} \frac{D P^{2} / 2}{D t} \simeq & \frac{1}{\rho_{0} c_{0}^{2}} \frac{\partial p_{1}^{2} / 2}{D t}-p_{0} \nabla \cdot \vec{u}_{1} \\
& +\frac{\gamma-1}{\gamma}\left(1-\frac{p_{1}}{p_{0}}\right)\left(q_{1}+\nabla \cdot\left(\lambda \vec{\nabla} T_{1}\right)+\overrightarrow{\vec{\tau}}_{1}: \vec{\nabla} \vec{u}_{1}\right)
\end{aligned}
$$

Moreover, approximating the total derivative of $\vec{u}^{2}$ by its partial derivative and neglecting higher order terms, the following third order approximation of the first term of Eq. 6 can be obtained easily:

$$
\rho \frac{D\left(\vec{u}^{2} / 2\right)}{D t} \approx \rho_{0} \frac{\partial\left(\vec{u}_{1}^{2} / 2\right)}{\partial t}
$$

The mean pressure $P_{0}$ being constant over space and the mean velocity being $u_{0}=0$, the following expression holds for the flux term of Eq. 6:

$$
\nabla \cdot(P \vec{u})=p_{0} \nabla \cdot \vec{u}_{1}+\nabla \cdot\left(p_{1} \vec{u}_{1}\right)
$$

Finally, injecting Eqs. 9, 10 and 11 into Eq. 6, the following equation is derived:

$$
\frac{\partial e_{1}}{\partial t}+\nabla \cdot\left(p_{1} \vec{u}_{1}\right)=\frac{\gamma-1}{\gamma p_{0}}\left(q_{1}+\nabla \cdot\left(\lambda \vec{\nabla} T_{1}\right)\right) p_{1}+\vec{u}_{1} \cdot\left(\vec{\nabla} \cdot \overrightarrow{\vec{\tau}}_{1}\right)
$$


which is a conservation equation for the classical acoustic energy:

$$
e_{1}=\rho_{0} \vec{u}_{1}^{2} / 2+\frac{1}{\rho_{0} c_{0}^{2}} p_{1}^{2} / 2
$$

Neglecting the viscous terms gives the classical equation for the acoustic energy [3]:

$$
\frac{\partial e_{1}}{\partial t}+\nabla \cdot\left(p_{1} \vec{u}_{1}\right)=\frac{\gamma-1}{\gamma p_{0}} p_{1} q_{1}
$$

The expected Rayleigh term $\left(p_{1} q_{1}\right)$ appears as a source term on the RHS of this equation. The first obvious observation is that the acoustic energy growth rate depends on the Rayleigh term but also on the acoustic fluxes $\nabla \cdot\left(p_{1} \vec{u}_{1}\right)$ so that, according to Eq. 14, the Rayleigh criterion is only a necessary condition for instability to occur. If Eq. 14 is integrated over the whole combustor $\Omega$, a more proper instability criterion is obtained stating that the combustor will oscillate (the total acoustic energy will grow) if:

$$
\iiint_{\Omega} \frac{\gamma-1}{\gamma p_{0}} p_{1} q_{1} d \Omega>\iint_{\Sigma} p_{1} \vec{u}_{1} d \Sigma
$$

or, in other words, if the source term due to combustion is larger than the acoustic losses on the combustor inlets and outlets surface $\Sigma$. The practical implication of Eq. (15) is that the classical Rayleigh criterion should not be used alone but should include also acoustic losses. This task is difficult in experiments since it requires the evaluation of acoustic fluxes $p_{1} \vec{u}_{1}$ on the boundaries of the burner but it can be done in LES. A first closure of Eq. 14 was performed for the first time in a self-excited combustor in 2004 by Martin et al [14] and showed similar order of magnitudes for the Rayleigh term and the acoustic losses.

Since no assumption has been made for defining the small amplitude fluctuations (except that they are indeed small compared to the characteristic scales), Eq. 12 describes a priori the evolution of the energy associated to the three classical types of disturbances put together (acoustic, entropy, vorticity). However, as a direct consequence of the zero Mach number assumption, the pulsation $\omega^{1}$ associated with the entropy and vortical modes is zero, corresponding to spatial disturbances which do not oscillate over time. Thus the present analysis essentially deals with acoustic perturbations. However, we shall see in the next section that the fluctuating flow is not isentropic and that an extended energy form is required.

\footnotetext{
1 indeed $\omega=k u_{0}$ where $k$ is the wavenumber of the perturbation and $u_{0}=0$ under the zero Mach number assumption.
} 


\section{The fluctuation energy (FE) equation}

\subsection{Why the AE (acoustic energy) form should not be used in flames}

Eq. 12 is a valid equation for $e_{1}$ but there is no evidence that this is the relevant quantity to describe the level of fluctuations in a turbulent reacting flow. Actually, the following simple example suggests that $e_{1}$ is not an appropriate measure of the fluctuation activity in a non-isentropic flow. Let us consider an hypothetical flow which initially contains only entropic linear fluctuations but no acoustic waves. The amount of AE associated with this "Flow 1" would be exactly zero since no velocity/pressure fluctuations are associated with the entropy mode (see Eq. 13). Hence "Flow 1" would be given the same amount of energy as the corresponding steady flow. A more disturbing observation is that, taking Flow 1 as an initial condition, it is easy to show that $e_{1}$ would increase as soon as the diffusivity $\lambda$ is not zero, even in the absence of combustion: indeed, in this simple non-reacting but initially non-isentropic flow, Eq. 7 shows that $p_{1}$ cannot remain zero since $\nabla \cdot\left(\lambda \vec{\nabla} T_{1}\right) \neq 0$ if an entropy fluctuation exists initially. Hence $e_{1}$ increases at time $t=0$ while the amount of fluctuations present in the flow necessarily decreases due to diffusivity. This result is clearly not satisfactory and shows that the classical acoustic energy defined by Eq. 13 is inadequate in a non-isentropic flow. In his paper, Chu [4] gave a tentative definition of what should be a fluctuation energy in such flows and insisted that it must decrease when viscosity/diffusivity effects cannot be neglected: this definition obviously does not apply to the AE form of Eq. 13. The fact that the quantity $e_{1}$, which comes from classical acoustic theory, is not relevant to purely entropic fluctuations is not very surprising. This means however that another quantity should be used in the case of reacting flows in order to characterise the global amount of fluctuations properly and that this energy should include entropy fluctuations.

\subsection{An extended fluctuation energy}

A natural way to proceed is then to start from Eq. 6 and combine it with an equation for entropy. Starting from the Gibbs equation:

$$
T d s=C_{v} d T-\frac{P}{\rho^{2}} d \rho=d e_{s}-\frac{P}{\rho^{2}} d \rho
$$


and using the continuity equation, the state equation $(p=\rho r T)$ as well as Eq. 4 leads to:

$$
\frac{D s}{D t}=\frac{r}{P}(q+\nabla \cdot(\lambda \vec{\nabla} T)+\overrightarrow{\vec{\tau}}: \overrightarrow{\vec{\nabla}} \vec{u})
$$

Multiplying Eq. 17 by $P s / r C_{p}$ and adding it to Eq. 6 directly gives:

$$
\begin{aligned}
\rho \frac{D \vec{u}^{2} / 2}{D t}+ & \frac{1}{\rho c^{2}} \frac{D P^{2} / 2}{D t}+\frac{P}{r C_{p}} \frac{D s^{2} / 2}{D t}+\nabla \cdot(P \vec{u})= \\
& \frac{s+r}{C_{p}}(q+\nabla \cdot(\lambda \vec{\nabla} T)+\overrightarrow{\vec{\tau}}: \overrightarrow{\vec{\nabla}} \vec{u})+\vec{u} \cdot(\vec{\nabla} \cdot \overrightarrow{\vec{\tau}})
\end{aligned}
$$

This equation is exact and can be linearized like Eq. 6 in section 2.2. Suppose that an entropy perturbation of small amplitude $s_{1}$ is super-imposed to the mean flow $s_{0}$. Then, since $D s_{0} / D t=\vec{u}_{1} \cdot \vec{\nabla} s_{0}$, Eq. 17 gives

$$
\frac{D s_{1}}{D t}=\frac{r}{P}\left(q_{1}+\nabla \cdot\left(\lambda \vec{\nabla} T_{1}\right)+\overrightarrow{\vec{\tau}}_{1}: \overrightarrow{\vec{\nabla}} \vec{u}_{1}\right)-\vec{u}_{1} \cdot \vec{\nabla} s_{0}
$$

so that, with

$$
\frac{D s^{2} / 2}{D t}=\frac{D s_{1}^{2} / 2}{D t}+\frac{D s_{1} s_{0}}{D t}+\frac{D s_{0}^{2} / 2}{D t}
$$

the following equation holds to third order:

$$
\frac{P}{r C_{p}} \frac{D s^{2} / 2}{D t} \simeq \frac{P_{0}}{r C_{p}} \frac{\partial s_{1}^{2} / 2}{\partial t}+\frac{1}{C_{p}}\left(q_{1}+\nabla \cdot\left(\lambda \vec{\nabla} T_{1}\right)+\overrightarrow{\vec{\tau}}_{1}: \vec{\nabla} \vec{u}_{1}\right)+\frac{P_{0}}{r C_{p}} s_{1} \vec{u}_{1} \cdot \vec{\nabla} s_{0}(21)
$$

The other terms of the LHS of Eq.18 have already been linearized in section 2.2 and, keeping only the second order terms, the linearized form of Eq. 18 becomes an equation for the fluctuation energy (FE equation) $e_{\text {tot }}$ :

$$
\begin{aligned}
\frac{\partial e_{\mathrm{tot}}}{\partial t}+\nabla \cdot\left(p_{1} \vec{u}_{1}\right) & =\frac{T_{1}}{T_{0}}\left(q_{1}+\nabla \cdot\left(\lambda \vec{\nabla} T_{1}\right)\right) \\
& -\frac{P_{0}}{r C_{p}} s_{1} \vec{u}_{1} \cdot \vec{\nabla} s_{0}+\vec{u}_{1} \cdot\left(\vec{\nabla} \cdot \overrightarrow{\vec{\tau}}_{1}\right)
\end{aligned}
$$

where $e_{\text {tot }}$ is defined by:

$$
e_{\text {tot }}=\rho_{0} \vec{u}_{1}^{2} / 2+\frac{1}{\rho_{0} c_{0}^{2}} p_{1}^{2} / 2+\frac{P_{0}}{r C_{p}} s_{1}^{2} / 2
$$


Equation 22 was first derived by Chu [4] except for the term in $\vec{\nabla} s_{0}$ which vanishes in the case where the mean flow entropy $s_{0}$ is uniform over space. It generalizes the classical acoustic energy (AE) form (Eq. 6) to the case of entropy/acoustic fluctuations and degenerates naturally to it in isentropic flows. Note that, in the general case, this FE (fluctuation energy) form must integrate the fluctuations of three variables $p_{1}, u_{1}$ and $s_{1}$ while the acoustic energy $e_{1}$ included only two. ${ }^{2}$ The fact that entropy is present in this extended energy does not mean that the entropy mode of fluctuation has been added to the analysis. Indeed, the zero Mach number was still used for the linearization process leading to the extended form Eq. 23. This means that only acoustic fluctuations are oscillating over time because the entropy and vortical modes correspond to the null frequency. However, Eq. 19 shows that the acoustic modes are not isentropic when unsteady heat release and/or mean entropy gradient are present. The extended form of the fluctuation energy is a natural way of accounting for this property.

Integrated over space, this equation suggests that the classical Rayleigh criterion $\iiint_{V} p_{1} q_{1} d V$ should be replaced by $\iiint_{V} T_{1} q_{1} d V$ in order to characterize the stability of a combustor. Specificaly, the global fluctuation energy in the system grows when (neglecting viscous effects):

$$
\iiint_{\Omega}\left(\frac{T_{1} q_{1}}{T_{0}}-\frac{P_{0}}{r C_{p}} s_{1} \vec{u}_{1} \cdot \vec{\nabla} s_{0}\right) d \Omega>\iint_{\Sigma} p_{1} \vec{u}_{1} \cdot \vec{n} d \Sigma
$$

This criterion extends the classical Rayleigh criterion to the case where the net energy flux at the boundaries cannot be neglected and the entropy fluctuations are significant. It also extends the initial equation of $\mathrm{Chu}$ to the case where the entropy field is not constant over space, which is always the case when combustion occurs. The second term of the LHS was not present in the Chu's paper [4] and does not seem to have been discussed earlier. It describes how the overall fluctuation energy decreases when a positive fluctuation of entropy $\left(s_{1}>0\right)$ is convected towards region with larger mean entropy $\left(\vec{u}_{1} \cdot \vec{\nabla} s_{0}>0\right)$, which is an expected result. A rough estimate of the order of magnitude of this term (without accounting for the phase shifts between the different terms) can be obtained as follows. Under the zero Mach number assumption, the mean pressure field $P_{0}$ is constant so that $\vec{\nabla} s_{0}=C_{p} / T_{0} \vec{\nabla} T_{0}$. Moreover, $\left|u_{1}\right| \propto\left|p_{1}\right| / \rho_{0} c_{0}$ for acoustic perturbations and, assuming that the main source of entropy fluctuations is the unsteady heat release, one obtains $\omega\left|s_{1}\right| \propto r\left|q_{1}\right| / P_{0}$, where $\omega$ is the pulsation. Introducing $L_{f}=\left|\vec{\nabla} T_{0}\right| / T_{0}$, the characteristic thickness of the flame brush, and $\lambda=2 \pi c_{0} / \omega$, the characteristic acoustic wavelength, the maximum (assuming optimal phase shifts) order of magnitude of the $\vec{\nabla} s_{0}$ term in Eq. 24 is given by $\left|p_{1}\right|\left|q_{1}\right| \times \lambda /\left(2 \pi(\gamma-1) L_{f}\right) \times(\gamma-1) / \gamma P_{0}$. The equivalent

2 Another expression for $e_{\text {tot }}$ is: $e_{\text {tot }}=\rho_{0} \vec{u}_{1}^{2} / 2+\frac{c_{0}^{2}}{\gamma \rho_{0}} \rho_{1}^{2} / 2+\frac{\rho_{0} C_{v}}{T_{0}} T_{1}^{2} / 2$. 
estimate of the classical Rayleigh term in Eq. 15 leads to $(\gamma-1)\left|p_{1}\right|\left|q_{1}\right| / \gamma P_{0}$. Since $L_{f}$ can be much smaller than $\lambda$, this means that the additional term related to the non uniform mean entropy field is potentially larger than the classical Rayleigh term. Specific post-processing of unsteady LES data will be necessary to address this issue more precisely.

More generally, the derivation of Eq. 24 shows how arbitrary the stability criteria can be since their form depends on the choice of the energy used to characterize the fluctuations. This issue is discussed in the next section.

\subsection{About the choice of an energy form}

At this point, two energies (Table 1) have been defined, leading to two different equations (Table 2) but also to different instability criteria (Eq. 15 and 24). Table 3 compares these criteria with the classical Rayleigh criterion in the simplest case where heat diffusivity and viscosity are both zero and mean entropy $s_{0}$ is constant. The criteria are also integrated with time over a period of the instability $\tau$. The first form (AE) leads to a stability criterion (Eq. 15) extending the Rayleigh criterion while the second one (FE) leads to a criterion (Eq. 24) which is very different. Interestingly, the AE Rayleigh criterion predicted instability when pressure and heat release fluctuations were in phase while the FE criterion requires temperature and heat release to be in phase.

A relevant question is then to determine which of these two forms is the most adequate. This can be done by pursuing the simple test case mentioned in Section 3.1: consider a domain with zero fluxes on boundaries and no combustion source term. A 'good' energy, according to Chu's definition[4] should only decrease in this situation and this decrease should be caused by dissipation. For simplification, the thermal diffusivity and molecular viscosity are assumed to be constant for this exercice and the gradients of the mean entropy $s_{0}$ and heat capacity ratio $\gamma$ are neglected. Starting from the equations of Table 2, integrating over the whole domain and setting $q_{1}$ and all boundary fluxes to zero leads to the following equations:

$$
\frac{\partial}{\partial t} \iiint_{\Omega} e_{1} d \Omega=-\lambda \frac{\gamma-1}{\gamma p_{0}} \iiint_{\Omega} \vec{\nabla} p_{1} \cdot \vec{\nabla} T_{1} d \Omega-\iiint_{\Omega} \overrightarrow{\vec{\tau}}_{1}: \overrightarrow{\vec{\nabla}} \vec{u}_{1} d \Omega
$$

and

$$
\frac{\partial}{\partial t} \iiint_{\Omega} e_{\text {tot }} d \Omega=-\frac{\lambda}{T_{0}} \iiint_{\Omega}\left(\vec{\nabla} T_{1}\right)^{2} d \Omega-\iiint_{\Omega} \overrightarrow{\vec{\tau}}_{1}: \overrightarrow{\vec{\nabla}} \vec{u}_{1} d \Omega
$$

The last term at the RHS is shared by both energy forms and is always negative 
since it is the classical dissipation function related to the velocity fluctuations. This means that both $e_{1}$ and $e_{\text {tot }}$ decrease when only viscosity is present. This is however not the case when only diffusivity is present and Eq. 25 and 26 provide a better understanding of Chu's criterion to choose an energy definition: if the flow is isentropic, pressure $\left(p_{1}\right)$ and temperature $\left(T_{1}\right)$ fluctuations are in phase and the RHS term of Eq. 25 is always negative so that the acoustic energy ( $\mathrm{AE}$ ) form $e_{1}$ is a proper estimate of energy. In all other cases, however, the RHS term of Eq. 25 can take any sign, increasing or decreasing the energy and making the $\mathrm{AE}$ form $e_{1}$ a quantity of limited interest. On the other hand, the RHS term of Eq. 26 is a truly dissipative term in all flows, even if they are not isentropic. This suggests that only the FE form of the energy should be used in flames. Whether this linearized form is valid in flames which exhibit very large entropy fluctuations remains to be checked. Post-processing of LES fields of compressible reacting turbulent flows will also be needed to estimate the magnitude of the different terms of the criterion derived for the FE form, Eq. 24. Specifically, such data would be necessary to precise the behavior of the $T_{1} q_{1}$ term with respect to its classical $p_{1} q_{1}$ counterpart as well as the magnitude of the term related to the mean entropy gradient.

\section{Conclusion}

This paper describes the construction of conservation equations for fluctuation energies in reacting flows. The AE (Acoustic Energy) form is first constructed starting from a non-linear energy equation. It is shown that the usual Rayleigh term is the source term of this equation but that another term (acoustic losses) plays a significant role in the budget of this equation. Second, it is shown that the $\mathrm{AE}$ form is insufficient to describe fluctuations in flames where entropy waves play a role. A new energy (FE for Fluctuation Energy) is defined and its conservation equation is derived. This equation shows that a different stability criterion is obtained in which temperature and heat release must be in phase to trigger the instability, while the Rayleigh criterion predicted instability when pressure and heat release are in phase. Another source term due to entropy gradients is also exhibited. These fundamental equations are believed to be building blocks to analyze results produced by recent compressible Large Eddy Simulations of turbulent flames in which all terms of the energy equations can be examined. On the long term, closing budgets of fluctuation energies in LES of unstable combustors could impact the understanding of combustion instabilities like budgets of turbulent kinetic energy did for turbulence near wall-bounded flows $[12,13]$ fifteen years ago. 


\section{Acknowledgments}

A significant part of this work was achieved during the 2004 Summer Program of the Center for Turbulence Research. Fruitful discussions with Pr S. Lele and J. Freund are gratefully acknowledged as well as the support of CTR and CERFACS for both authors. 


\section{Tables}

Table 1

\begin{tabular}{|c||c|}
\hline $\mathrm{AE}$ & $e_{1}=\rho_{0} \vec{u}_{1}^{2} / 2+\frac{1}{\rho_{0} c_{0}^{2}} p_{1}^{2} / 2$ \\
\hline $\mathrm{FE}$ & $e_{\text {tot }}=\rho_{0} \vec{u}_{1}^{2} / 2+\frac{1}{\rho_{0} c_{0}^{2}} p_{1}^{2} / 2+\frac{P_{0}}{r C_{p}} s_{1}^{2} / 2$ \\
\hline
\end{tabular}

Definitions of the Acoustic Energy (AE) and of the Fluctuation Energy (FE).

\begin{tabular}{|c||c|}
\hline AE & $\frac{\partial e_{1}}{\partial t}+\nabla \cdot\left(p_{1} \vec{u}_{1}\right)=\frac{\gamma-1}{\gamma p_{0}}\left(q_{1}+\nabla \cdot\left(\lambda \vec{\nabla} T_{1}\right)\right) p_{1}+\vec{u}_{1} \cdot\left(\vec{\nabla} \cdot \vec{\tau}_{1}\right)$ \\
\hline FE & $\frac{\partial e_{\text {tot }}}{\partial t}+\nabla \cdot\left(p_{1} \vec{u}_{1}\right)=\frac{T_{1}}{T_{0}}\left(q_{1}+\nabla \cdot\left(\lambda \vec{\nabla} T_{1}\right)\right)-\frac{P_{0}}{r C_{p}} s_{1} \vec{u}_{1} \cdot \vec{\nabla} s_{0}+\vec{u}_{1} \cdot\left(\vec{\nabla} \cdot \overrightarrow{\vec{\tau}}_{1}\right)$ \\
\hline
\end{tabular}

Conservation equations for the Acoustic Energy (AE) and Fluctuation Energy (FE).

Table 3

\begin{tabular}{|c||c|}
\hline Classical Rayleigh & $\iiint_{\Omega} p_{1} q_{1} d t d V>0$ \\
\hline Extended Rayleigh & $\frac{(\gamma-1)}{\gamma p_{0}} \iiint_{\Omega} p_{1} q_{1} d t d V>\iint_{\Sigma} p_{1} \vec{u}_{1} \cdot \vec{n} d \Sigma$ \\
\hline Chu & $\frac{1}{T_{0}} \iiint_{\Omega} T_{1} q_{1} d t d V>\iint_{\Sigma} p_{1} \vec{u}_{1} \cdot \vec{n} d \Sigma$ \\
\hline
\end{tabular}

Summary of criteria for combustion instability for zero thermal diffusivity, zero viscosity and constant mean entropy. These criteria should also be integrated over time but this integration is not indicated here for clarity. 


\section{References}

1. Landau, L. and Lifchitz, E., Physique Theorique - Tome 6 : Mecanique des Fluides. Ed. Librairie du Globe- Editions MIR, 1984.

2. Crighton, D. G., Dowling, A., Ffowcs Williams, J., Heckl, M. and Leppington, F., Modern methods in analytical acoustics. Springer Verlag, New-York, 1992.

3. Poinsot, T. and Veynante, D., Theoretical and numerical combustion. R.T. Edwards, 2001.

4. Chu, B. T., Acta Mechanica: 215-234 (1965).

5. Putnam, A. A., Combustion driven oscillations in industry. American Elsevier, 1971.

6. Williams, F. A., Combustion theory. Benjamin Cummings, Menlo Park, CA, 1985 .

7. Rayleigh, L., Nature July 18: 319-321 (1878).

8. Huang, Y., Sung, H. G., Hsieh, S. Y. and Yang, V., J. Prop. Power 19: 782-794 (2003).

9. Mahesh, K., Constantinescu, G. and Moin, P., J. Comput. Phys. 197: 215-240 (2004).

10. Pierce, C. D. and Moin, P., J. Fluid Mech. 504: 73-97 (2004).

11. Selle, L., Lartigue, G., Poinsot, T., Koch, R., Schildmacher, K.-U., Krebs, W., Prade, B., Kaufmann, P. and Veynante, D., Combust. Flame 137: 489-505 (2004).

12. Mansour, N., Kim, J. W. and Moin, P., J. Fluid Mech. 194: 15-44 (1988). 
13. Moin, P. and Mahesh, K., Ann. Rev. Fluid Mech. 30: 539578 (1998).

14. Martin, C., Benoit, L., Nicoud, F. and Poinsot, T., Proc of the Summer Program, p. 377-394, Center for Turbulence Research, NASA Ames/Stanford Univ., (2004). 